\title{
Suppression of superconductivity by inhomogeneous chiral condensation in the $\mathrm{NJL}_{2}$ model
}

\author{
D. Ebert ${ }^{1)}$, T.G. Khunjua ${ }^{2)}$, K.G. Klimenko ${ }^{3)}$, and V.Ch. Zhukovsky ${ }^{2)}$ \\ 1) Institute of Physics, Humboldt-University Berlin, 12489 Berlin, Germany \\ 2) Faculty of Physics, Moscow State University, 119991, Moscow, Russia and \\ 3) IHEP and University "Dubna" (Protvino branch), 142281, Protvino, Moscow Region, Russia
}

\begin{abstract}
We investigate the possibility of spatially inhomogeneous chiral and Cooper, or superconducting, pairing in the (1+1)-dimensional model by Chodos et al. [ Phys. Rev. D61, 045011 (2000)] generalized to continuous chiral invariance. The consideration is performed at nonzero values of temperature $T$ and quark number chemical potential $\mu$. In the framework of the Fulde-Ferrel inhomogeneity ansatz for chiral and Cooper condensates, we show that if $G_{1}>G_{2}$, where $G_{1}$ and $G_{2}$ are the coupling constants in the quark-antiquark and diquark channels, then in the $(\mu, T)$-phase diagram the superconducting phase is suppressed by spatially inhomogeneous chiral spiral phase with broken chiral symmetry. In contrast, in the above mentioned original Chodos et al. model, where only the opportunity for homogeneous condensates to be formed is taken into account, the superconducting phase is realized at sufficiently high values of $\mu$ at arbitrary values of $G_{2}>0$, including the interval $0<G_{2}<G_{1}$.
\end{abstract}

\section{INTRODUCTION}

In the last years great attention was devoted to the investigation of dense quark (or baryonic) matter. The interest is motivated by possible existence of quark matter inside compact stars or its creation at heavy ion collisions. In nature as well as in heavy ion collision experiments, the quark matter densities are not high enough, so consideration of its properties is not possible in the framework of perturbation approximation of weak coupling QCD. Usually, various effective theories such as Nambu - Jona-Lasinio (NJL) model, $\sigma$-model etc. are more adequate for studying the QCD and quark matter phase diagram in this case. As a result, a variety of spatially non-uniform (inhomogeneous) quark matter phases related to chiral symmetry breaking, color superconductivity, and charged pion condensation phenomenon etc. were predicted (see, e.g., 1 9], and references therein) at rather low values of temperature and baryon density.

The phenomenon of spatially non-uniform quark pairing was also intensively investigated within different $(1+1)$ dimensional toy models which can mimic qualitatively the QCD phase diagram. In this connection, it is necessary to mention the Gross-Neveu (GN) type models that are symmetrical with respect to the discrete and continuous chiral transformations and extended by inclusion of baryon and isospin chemical potentials. In the framework of these models both the inhomogeneous chiral [10 12] and charged pion condensation phenomena were considered [13 15]. (In order to overcome the no-go theorem for spontaneous breaking of continuous symmetry in (1+1)-dimensions, consideration there was performed in the large- $N$ approximation, where $N$ is the number of quark multiplets.) Moreover, it is necessary to note that in some quasi-one-dimensional condensed matter systems, such as imbalanced Fermi gases [16], etc., there might exist different inhomogeneous phases.

Among a variety of GN-type models, there is one which describes competition between quark-antiquark and diquark pairing [17]. In [17], the consideration is performed in the supposition that chiral and superconducting condensates are spatially homogeneous. In this case it was shown that if $G_{1}>G_{2}$, where $G_{1}$ and $G_{2}$ are the coupling constants in the chiral and superconducting channels, respectively, then at rather high quark number chemical potential the superconducting phase is realized in the system. However, the condensates in the genuine ground state of the model may be inhomogeneous, and hence, the aim of our paper is to investigate such a possibility. Namely, we study the phase structure of the extended model [17] (which is symmetric with respect to continuous chiral transformations), assuming that both quark-antiquark and diquark condensates might have a spatial inhomogeneity in the form of the Fulde-Ferrel single plane wave ansatz [18], for simplicity. As a result, we have shown that, in contrast to a homogeneous ansatz for condensates, at $G_{1}>G_{2}$ superconductivity is absent and only inhomogeneous chiral spiral (or chiral density wave) phase occurs at arbitrary $\mu>0$ (for rather low temperature).

\section{THE MODEL AND ITS EFFECTIVE ACTION}

Our investigation is based on a (1+1)-dimensional $\mathrm{NJL}_{2}$-type model with massless fermions, or quarks, belonging to a fundamental multiplet of the $O(N)$ flavor group. Its Lagrangian describes the interaction in the quark-antiquark as well as in the scalar diquark channels:

$$
L=\sum_{k=1}^{N} \bar{\psi}_{k}\left[\gamma^{\nu} i \partial_{\nu}+\mu \gamma^{0}\right] \psi_{k}+\frac{G_{1}}{N}\left[\left(\sum_{k=1}^{N} \bar{\psi}_{k} \psi_{k}\right)^{2}+\left(\sum_{k=1}^{N} \bar{\psi}_{k} i \gamma^{5} \psi_{k}\right)^{2}\right]+\frac{G_{2}}{N}\left(\sum_{k=1}^{N} \psi_{k}^{T} \epsilon \psi_{k}\right)\left(\sum_{j=1}^{N} \bar{\psi}_{j} \epsilon \bar{\psi}_{j}^{T}\right)
$$


where $\mu$ is the quark number chemical potential. As it is noted above, all fermion fields $\psi_{k}(k=1, \ldots, N)$ form a fundamental multiplet of $O(N)$ group. Moreover, each field $\psi_{k}$ is a two-component Dirac spinor (the symbol $T$ denotes the transposition operation). The quantities $\gamma^{\nu}(\nu=0,1), \gamma^{5}$, and $\epsilon$ in (1) are matrices in the spinor space,

$$
\gamma^{0}=\left(\begin{array}{cc}
0 & 1 \\
1 & 0
\end{array}\right) ; \quad \gamma^{1}=\left(\begin{array}{cc}
0 & -1 \\
1 & 0
\end{array}\right) \equiv-\epsilon ; \quad \gamma^{5}=\gamma^{0} \gamma^{1}=\left(\begin{array}{cc}
1 & 0 \\
0 & -1
\end{array}\right) .
$$

Clearly, the Lagrangian $L$ is invariant under transformations from the internal $O(N)$ group, which is introduced here in order to make it possible to perform all the calculations in the framework of the nonperturbative large- $N$ expansion method. Physically more interesting is that the model (1) is invariant under transformations from the $U_{V}(1)$ quark number group: $\psi_{k} \rightarrow \exp (i \alpha) \psi_{k}(k=1, \ldots, N)$. In addition, the Lagrangian is invariant under the continuous group $U_{A}(1)$ of chiral transformations: $\psi_{k} \rightarrow \exp \left(i \alpha^{\prime} \gamma^{5}\right) \psi_{k}(k=1, \ldots, N)$. 1 The linearized version of Lagrangian (11) that contains auxiliary scalar bosonic fields $\sigma(x), \pi(x), \Delta(x), \Delta^{*}(x)$ has the following form

$$
\mathcal{L}=\bar{\psi}_{k}\left[\gamma^{\nu} i \partial_{\nu}+\mu \gamma^{0}-\sigma-i \gamma^{5} \pi\right] \psi_{k}-\frac{N}{4 G_{1}}\left(\sigma^{2}+\pi^{2}\right)-\frac{N}{4 G_{2}} \Delta^{*} \Delta-\frac{\Delta^{*}}{2}\left[\psi_{k}^{T} \epsilon \psi_{k}\right]-\frac{\Delta}{2}\left[\bar{\psi}_{k} \epsilon \bar{\psi}_{k}^{T}\right]
$$

(Here and in what follows summation over repeated indices $k=1, \ldots, N$ is implied.) Clearly, the Lagrangians (1) and (3) are equivalent, as can be seen by using the Euler-Lagrange equations of motion for scalar bosonic fields, which take the form

$$
\sigma(x)=-2 \frac{G_{1}}{N}\left(\bar{\psi}_{k} \psi_{k}\right), \quad \pi(x)=-2 \frac{G_{1}}{N}\left(\bar{\psi}_{k} i \gamma^{5} \psi_{k}\right), \quad \Delta(x)=-2 \frac{G_{2}}{N}\left(\psi_{k}^{T} \epsilon \psi_{k}\right), \quad \Delta^{*}(x)=-2 \frac{G_{2}}{N}\left(\bar{\psi}_{k} \epsilon \bar{\psi}_{k}^{T}\right) .
$$

One can easily see from (4) that the (neutral) fields $\sigma(x)$ and $\pi(x)$ are real quantities, i.e. $(\sigma(x))^{\dagger}=\sigma(x),(\pi(x))^{\dagger}=$ $\pi(x)$ (the superscript symbol $\dagger$ denotes the Hermitian conjugation), but the (charged) diquark scalar fields $\Delta(x)$ and $\Delta^{*}(x)$ are mutually Hermitian conjugated complex quantities, so that $(\Delta(x))^{\dagger}=\Delta^{*}(x)$ and vice versa. Clearly, all the fields (4) are singlets with respect to the $O(N)$ group. 2 If the scalar diquark field $\Delta(x)$ has a nonzero ground state expectation value, i.e. $\langle\Delta(x)\rangle \neq 0$, the Abelian quark number $U_{V}(1)$ symmetry of the model is spontaneously broken down. However, if $\langle\sigma(x)\rangle \neq 0$ then the continuous $U_{A}(1)$ chiral symmetry of the model is spontaneously broken.

Let us now study the phase structure of the four-fermion model (1) by starting with the equivalent semi-bosonized Lagrangian (3). In the leading order of the large- $N$ approximation, the effective action $\mathcal{S}_{\text {eff }}\left(\sigma, \pi, \Delta, \Delta^{*}\right)$ of the considered model is expressed in terms of the path integral over fermion fields:

$$
\exp \left(i \mathcal{S}_{\text {eff }}\left(\sigma, \pi, \Delta, \Delta^{*}\right)\right)=\int \prod_{l=1}^{N}\left[d \bar{\psi}_{l}\right]\left[d \psi_{l}\right] \exp \left(i \int \mathcal{L} d^{2} x\right)
$$

where

$$
\mathcal{S}_{\text {eff }}\left(\sigma, \pi, \Delta, \Delta^{*}\right)=-\int d^{2} x\left[\frac{N}{4 G_{1}}\left(\sigma^{2}(x)+\pi^{2}(x)\right)+\frac{N}{4 G_{2}} \Delta(x) \Delta^{*}(x)\right]+\widetilde{\mathcal{S}}_{\text {eff }}
$$

The fermion contribution to the effective action, i.e. the term $\widetilde{\mathcal{S}}_{\text {eff }}$ in (5), is given by:

$$
\exp \left(i \widetilde{\mathcal{S}}_{\text {eff }}\right)=\int \prod_{l=1}^{N}\left[d \bar{\psi}_{l}\right]\left[d \psi_{l}\right] \exp \left\{i \int\left[\bar{\psi}_{k}\left(\gamma^{\nu} i \partial_{\nu}+\mu \gamma^{0}-\sigma-i \gamma^{5} \pi\right) \psi_{k}-\frac{\Delta^{*}}{2}\left(\psi_{k}^{T} \epsilon \psi_{k}\right)-\frac{\Delta}{2}\left(\bar{\psi}_{k} \epsilon \bar{\psi}_{k}^{T}\right)\right] d^{2} x\right\} .
$$

The ground state expectation values $\langle\sigma(x)\rangle,\langle\pi(x)\rangle$, etc of the composite bosonic fields are determined by the saddle point equations,

$$
\frac{\delta \mathcal{S}_{\mathrm{eff}}}{\delta \sigma(x)}=0, \quad \frac{\delta \mathcal{S}_{\mathrm{eff}}}{\delta \pi(x)}=0, \quad \frac{\delta \mathcal{S}_{\mathrm{eff}}}{\delta \Delta(x)}=0, \quad \frac{\delta \mathcal{S}_{\mathrm{eff}}}{\delta \Delta^{*}(x)}=0
$$

In vacuum, i.e. in the state corresponding to an empty space with zero particle density and zero value of the chemical potential $\mu$, the above mentioned quantities $\langle\sigma(x)\rangle$, etc. (17) do not depend on space coordinates. However, in a dense medium, when $\mu \neq 0$, the ground state expectation values of bosonic fields (4) might have a nontrivial dependence on the spatial coordinate $x$. For simplicity, in this paper we will use the following well-known ansatz:

$$
\langle\sigma(x)\rangle=M \cos (2 b x), \quad\langle\pi(x)\rangle=M \sin (2 b x), \quad\langle\Delta(x)\rangle=\Delta \exp \left(-2 i b^{\prime} x\right), \quad\left\langle\Delta^{*}(x)\right\rangle=\Delta \exp \left(2 i b^{\prime} x\right),
$$

\footnotetext{
${ }^{1}$ Earlier in [17], the similar model symmetric under discrete $\gamma^{5}$ chiral transformation was investigated. However, only the possibility for the spatially homogeneous chiral and diquark condensates was considered there. In our paper the model by Chodos et al. 17] is generalized to the case of continuous chiral invariance in order to study the inhomogeneous chiral condensates in the form of chiral spirals (or chiral density waves).

${ }^{2}$ Note that the $\Delta(x)$ field is a flavor $\mathrm{O}(\mathrm{N})$ singlet, since the representations of this group are real.
} 
where $M, b, b^{\prime}$ and $\Delta$ are real constant quantities. (It means that we suppose for $\langle\sigma(x)\rangle$ and $\langle\pi(x)\rangle$ the chiral spiral (or chiral density wave) ansatz, and the Fulde-Ferrel [18] single plane wave one for diquark condensates.) In fact, $M, b, b^{\prime}$ and $\Delta$ are coordinates of the global minimum point of the thermodynamic potential (TDP) $\Omega\left(M, b, b^{\prime}, \Delta\right)$. 3 In the leading order of the large- $N$ expansion it is defined by the following expression:

$$
\int d^{2} x \Omega\left(M, b, b^{\prime}, \Delta\right)=-\left.\frac{1}{N} \mathcal{S}_{\text {eff }}\left\{\sigma(x), \pi(x), \Delta(x), \Delta^{*}(x)\right\}\right|_{\sigma(x)=\langle\sigma(x)\rangle, \pi(x)=\left\langle\pi_{a}(x)\right\rangle, \ldots},
$$

which gives

$$
\begin{aligned}
\int d^{2} x \Omega\left(M, b, b^{\prime}, \Delta\right)= & \int d^{2} x\left(\frac{M^{2}}{4 G_{1}}+\frac{\Delta^{2}}{4 G_{2}}\right)+\frac{i}{N} \ln \left(\int \prod _ { l = 1 } ^ { N } [ d \overline { \psi } _ { l } ] [ d \psi _ { l } ] \operatorname { e x p } \left(i \int d ^ { 2 } x \left[\bar{\psi}_{k} \mathcal{D} \psi_{k}\right.\right.\right. \\
& \left.\left.\left.-\frac{\Delta \exp \left(2 i b^{\prime} x\right)}{2}\left(\psi_{k}^{T} \epsilon \psi_{k}\right)-\frac{\Delta \exp \left(-2 i b^{\prime} x\right)}{2}\left(\bar{\psi}_{k} \epsilon \bar{\psi}_{k}^{T}\right)\right]\right)\right),
\end{aligned}
$$

where $\mathcal{D}=\gamma^{\rho} i \partial_{\rho}+\mu \gamma^{0}-M \exp \left(2 i \gamma^{5} b x\right)$. To proceed, let us introduce in (9) the new fermion fields, $q_{k}=\exp \left[i\left(b^{\prime}+\right.\right.$ $\left.\left.\gamma^{5} b\right) x\right] \psi_{k}$ and $\bar{q}_{k}=\bar{\psi}_{k} \exp \left[i\left(\gamma^{5} b-b^{\prime}\right) x\right]$. Since this transformation of fermion fields does not change the path integral measure in (9) 4, the expression (9) for the thermodynamic potential is easily transformed into the following one:

$$
\begin{aligned}
\int d^{2} x \Omega\left(M, b, b^{\prime}, \Delta\right) & =\int d^{2} x\left(\frac{M^{2}}{4 G_{1}}+\frac{\Delta^{2}}{4 G_{2}}\right) \\
& +\frac{i}{N} \ln \left(\int \prod_{l=1}^{N}\left[d \bar{q}_{l}\right]\left[d q_{l}\right] \exp \left(i \int d^{2} x\left[\bar{q}_{k} D q_{k}-\frac{\Delta}{2}\left(q_{k}^{T} \epsilon q_{k}\right)-\frac{\Delta}{2}\left(\bar{q}_{k} \epsilon \bar{q}_{k}^{T}\right)\right]\right)\right),
\end{aligned}
$$

where

$$
D=\gamma^{\nu} i \partial_{\nu}+(\mu-b) \gamma^{0}-M+\gamma^{1} b^{\prime} .
$$

The path integration in this expression can be evaluated (see, e.g., Appendix B of the paper [20]), so we have for the TDP

$$
\Omega\left(M, b, b^{\prime}, \Delta\right) \equiv \Omega^{u n}\left(M, b, b^{\prime}, \Delta\right)=\frac{M^{2}}{4 G_{1}}+\frac{\Delta^{2}}{4 G_{2}}+\frac{i}{2} \int \frac{d^{2} p}{(2 \pi)^{2}} \ln \left(\lambda_{1}(p) \lambda_{2}(p)\right),
$$

where $(\tilde{\mu}=\mu-b)$

$$
\lambda_{1,2}(p)=p_{0}^{2}-\tilde{\mu}^{2}-p_{1}^{2}+b^{\prime 2}+M^{2}-\Delta^{2} \pm 2 \sqrt{M^{2} p_{0}^{2}-M^{2} p_{1}^{2}+\tilde{\mu}^{2} p_{1}^{2}-2 p_{0} b^{\prime} \tilde{\mu} p_{1}+p_{0}^{2} b^{\prime 2}}
$$

and superscription "un" denotes the unrenormalized quantity. In the following we will study the behavior of the global minimum point of this TDP as a function of dynamical variables $M, b, b^{\prime}, \Delta$ vs the external parameter $\mu$ in two qualitatively different cases: i) The case of homogeneous condensates, i.e. when in (12) both $b$ and $b^{\prime}$ are supposed to be put to zero by hand, i.e. without any proof, ii) The case of spatially inhomogeneous condensates, i.e. when the quantities $b$ and $b^{\prime}$ are defined dynamically by the gap equations of the TDP (12). Note finally that the expression (12) is the TDP of the initial system at zero temperature $T$. The consideration of the case $T \neq 0$ will also be taken into account in the subsequent sections.

\section{HOMOGENEOUS ANSATZ FOR BOTH CONDENSATES: $b=0, b^{\prime}=0$}

In this section we assume both chiral and superconducting condensates to be homogeneous, i.e. putting $b=0$ and $b^{\prime}=0$. Then the TDP (12) is reduced to the following expression

$$
\Omega^{u n}(M, \Delta)=\frac{M^{2}}{4 G_{1}}+\frac{\Delta^{2}}{4 G_{2}}+\frac{i}{2} \int \frac{d^{2} p}{(2 \pi)^{2}} \ln \left[\left(p_{0}^{2}-E_{+}^{2}\right)\left(p_{0}^{2}-E_{-}^{2}\right)\right],
$$

where (note, $E_{-}^{2}$ is a nonnegative quantity)

$$
E_{ \pm}^{2}=\mu^{2}+p_{1}^{2}+M^{2}+\Delta^{2} \pm 2 \sqrt{M^{2} \Delta^{2}+\mu^{2}\left(p_{1}^{2}+M^{2}\right)} .
$$

\footnotetext{
${ }^{3}$ Here and in what follows we will use a rather conventional notation "global" minimum in the sense that among all our numerically found local minima the thermodynamical potential takes in their case the lowest value. This does not exclude the possibility that there exist other inhomogeneous condensates, different from [8), which lead to ground states with even lower values of the TDP.

4 This nontrivial fact follows from the investigations by Fujikawa [19], who established that a chiral transformation of spinor fields changes the path integral measure only in the case, when there is interaction between spinor and gauge fields.
} 
Integrating in (14) over $p_{0}$ (see Ref. [21] for similar integrals), one obtains for the TDP $\Omega^{u n}(M, \Delta)$ :

$$
\Omega^{u n}(M, \Delta)=\frac{M^{2}}{4 G_{1}}+\frac{\Delta^{2}}{4 G_{2}}-\int_{-\infty}^{\infty} \frac{d p_{1}}{4 \pi}\left(E_{+}+E_{-}\right) .
$$

Clearly, without loss of generality one can study the TDP (14)-(16) in the region $M \geq 0$ and $\Delta \geq 0$ at $\mu \geq 0$.

\section{A. The renormalization}

Formally, the TDP (14)-(16) is an ultraviolet divergent quantity. To renormalize it, i.e. to obtain a finite expression for it, we first need to regularize the TDP (16) by cutting off its integration region, $\left|p_{1}\right|<\Lambda$ :

$$
\Omega^{u n}(M, \Delta) \rightarrow \Omega^{r e g}(M, \Delta)=\frac{M^{2}}{4 G_{1}}+\frac{\Delta^{2}}{4 G_{2}}-\int_{0}^{\Lambda} \frac{d p_{1}}{2 \pi}\left(E_{+}+E_{-}\right) .
$$

Second, we must find such dependencies of the bare coupling constants $G_{1} \equiv G_{1}(\Lambda)$ and $G_{2} \equiv G_{2}(\Lambda)$ vs $\Lambda$ that in the limit $\Lambda \rightarrow \infty$ one can obtain from $\Omega^{r e g}(M, \Delta)$ a finite expression. To get the quantities $G_{1}(\Lambda)$ and $G_{2}(\Lambda)$ let us first use in (17) the following asymptotic expansion at $p_{1} \rightarrow \infty$ :

$$
E_{+}+E_{-}=2 E+\frac{\Delta^{2}}{E}+\mathcal{O}\left(1 / E^{2}\right)
$$

where $E=\sqrt{M^{2}+p_{1}^{2}}$. Then, substituting (18) into (17) and integrating there over $p_{1}$, we obtain the following asymptotic expansion of the regularized TDP at $\Lambda \rightarrow \infty$ :

$$
\Omega^{r e g}(M, \Delta)=\frac{M^{2}}{4 G_{1}}+\frac{\Delta^{2}}{4 G_{2}}-\frac{1}{2 \pi}\left(\Lambda^{2}+\left(M^{2}+\Delta^{2}\right) \ln \frac{\Lambda+\sqrt{\Lambda^{2}+M^{2}}}{M}\right)+\mathcal{O}\left(\Lambda^{0}\right) .
$$

Now it is obvious from (19) that all ultraviolet divergences (up to unessential term $-\Lambda^{2} / 2 \pi$ ) of the thermodynamic potential (17) can be removed, if

$$
\frac{1}{4 G_{1}} \equiv \frac{1}{4 G_{1}(\Lambda)}=\frac{1}{2 \pi} \ln \frac{2 \Lambda}{M_{1}}, \quad \frac{1}{4 G_{2}} \equiv \frac{1}{4 G_{2}(\Lambda)}=\frac{1}{2 \pi} \ln \frac{2 \Lambda}{M_{2}},
$$

where $M_{1}$ and $M_{2}$ are some finite and cutoff independent parameters of the model. In addition, $M_{1}$ and $M_{2}$ are also renormalization invariant, i.e. they do not depend on normalization points. (The physical meaning of these parameters will be discussed below.) Hence, in the limit $\Lambda \rightarrow \infty$ one can obtain from (17) a finite renormalization invariant expression for the TDP

$$
\Omega(M, \Delta)=\lim _{\Lambda \rightarrow \infty}\left\{\left.\Omega^{r e g}(M, \Delta)\right|_{G_{1}=G_{1}(\Lambda), G_{2}=G_{2}(\Lambda)}+\frac{\Lambda^{2}}{2 \pi}\right\} .
$$

In the following, instead of treating the results in terms of two dimensional parameters $M_{1,2}$, we will use, as in the paper [17], one dimensional, $M_{1}$, and one dimensionless, $\delta$, parameters, i.e.

$$
\frac{\delta}{4 \pi} \equiv \frac{1}{4 G_{2}}-\frac{1}{4 G_{1}}=\frac{1}{2 \pi} \ln \frac{M_{1}}{M_{2}} .
$$

Since $M_{1}$ and $\delta$ might be considered as free model parameters, it is clear that the renormalization procedure of the $\mathrm{NJL}_{2}$ model (1) is accompanied by the partial dimensional transmutation phenomenon. Indeed, in the initial unrenormalized expression (14) for $\Omega^{u n}(M, \Delta)$ two dimensionless bare coupling constants $G_{1,2}$ are present, whereas after renormalization the thermodynamic potential (21) is characterized in our choice of a parameterization by one dimensional, $M_{1}$, and one dimensionless, $\delta$, free model parameters.

\section{B. The phase structure}

In this subsection we will study the phase structure of the model (1) in three cases, first at $\mu=0$ and $T=0$ (it is the vacuum case), second at $\mu>0, T=0$ and, finally, at $\mu>0$ and $T>0$. All the condensates are still supposed to be homogeneous.

The vacuum case $(\mu=0, T=0)$. Putting $\mu=0$ in (17), we have for the regularized effective potential $V_{0}^{r e g}(M, \Delta)$ ( TDP in vacuum is usually called effective potential) the following expression:

$$
V_{0}^{r e g}(M, \Delta)=\frac{M^{2}}{4 G_{1}}+\frac{\Delta^{2}}{4 G_{2}}-\int_{0}^{\Lambda} \frac{d p_{1}}{2 \pi}\left(\sqrt{p_{1}^{2}+(M+\Delta)^{2}}+\sqrt{p_{1}^{2}+(M-\Delta)^{2}}\right) .
$$


After integration in (23) over $p_{1}$ we should renormalize the obtained expression, i.e. to put $G_{1}=G_{1}(\Lambda), G_{2}=G_{2}(\Lambda)$ (see in (20) ) and then to find a limit at $\Lambda \rightarrow \infty$ of the expression (21). In terms of $M_{1}$ and $\delta$ the renormalized effective potential looks like

$$
4 \pi V_{0}(M, \Delta)=\delta \Delta^{2}-\Delta^{2}-M^{2}+(M-\Delta)^{2} \ln \left|\frac{M-\Delta}{M_{1}}\right|+(M+\Delta)^{2} \ln \left(\frac{M+\Delta}{M_{1}}\right) .
$$

If $\delta>0$, i.e., as is easily seen from (20) and (22), at $G_{1}>G_{2}$, the global minimum of the effective potential (24) lies at the point $\left(M=M_{1}, \Delta=0\right)$. This means that if interaction in the quark-antiquark channel is greater than in the diquark one, then the chiral symmetry $U_{A}(1)$ of the model is spontaneously broken down and fermions acquire dynamically a nonzero mass, which is equal just to the free model parameter $M_{1}$. However, if $\delta<0$, i.e. at $G_{1}<G_{2}$, then the global minimum of the effective potential (24) is arranged at the point $\left(M=0, \Delta=\Delta_{0}(\delta)\right)$, where $\Delta_{0}(\delta)=M_{1} \exp (-\delta / 2)=M_{2}$. Since in this case only the diquark condensate is nonzero, the fermion number $U_{V}(1)$ symmetry is spontaneously broken down.

The case $\mu>0$ and $T=0$. Obviously, at $\mu>0$ the regularized TDP (17) can be presented in the form

$$
\Omega^{r e g}(M, \Delta)=V_{0}^{r e g}(M, \Delta)-\int_{0}^{\Lambda} \frac{d p_{1}}{2 \pi}\left(E_{+}+E_{-}-\sqrt{p_{1}^{2}+(M+\Delta)^{2}}-\sqrt{p_{1}^{2}+(M-\Delta)^{2}}\right)
$$

where $V_{0}^{r e g}(M, \Delta)$ is given in (23). As a result, at $\Lambda \rightarrow \infty$ one can obtain from (25) and (21) the renormalized TDP

$$
\Omega(M, \Delta)=V_{0}(M, \Delta)-\int_{0}^{\infty} \frac{d p_{1}}{2 \pi}\left(E_{+}+E_{-}-\sqrt{p_{1}^{2}+(M+\Delta)^{2}}-\sqrt{p_{1}^{2}+(M-\Delta)^{2}}\right),
$$

where $V_{0}(M, \Delta)$ is presented in (24). After numerical investigations of the function (26), it is clear that its global minimum might lie only in the points of the form $(M \geq 0, \Delta=0)$ or $(M=0, \Delta \geq 0)$. As a result, for further consideration it is enough to reduce the TDP (26) to the $M$ - and $\Delta$-axes, where it looks like

$$
\begin{aligned}
& 4 \pi \Omega(M, \Delta=0)=M^{2}\left(\ln \frac{M^{2}}{M_{1}^{2}}-1\right)+2 \theta(\mu-M)\left[M^{2} \ln \frac{\mu+\sqrt{\mu^{2}-M^{2}}}{M}-\mu \sqrt{\mu^{2}-M^{2}}\right], \\
& 4 \pi \Omega(M=0, \Delta)=\delta \Delta^{2}+\Delta^{2}\left(\ln \frac{\Delta^{2}}{M_{1}^{2}}-1\right)-2 \mu^{2},
\end{aligned}
$$

respectively. Apart from a trivial extremum at the origin for both functions (27) and (28), the TDP (27) has a nontrivial extremum at the point $M=M_{1}$, whereas the second TDP, i.e. the function (28), has a nontrivial minimum point at $\Delta=M_{1} \exp (-\delta / 2)=M_{2}$. Comparing the values of the functions (27)-(28) at these extrema, we obtain the following evolution of the global minimum point (GMP) of the TDP (26) vs $\mu$ and, as a result, the phase portrait of the initial model.

First, let us suppose that $\delta>0$, i.e. $G_{1}>G_{2}$. Then at sufficiently low $\mu$-values the GMP of the TDP (26) lies at the point $\left(M=M_{1}, \Delta=0\right)$, which corresponds to the chiral symmetry breaking phase of the model. In contrast, at sufficiently high values of the chemical potential the GMP of the TDP (26) is arranged at the point $\left(M=0, \Delta=M_{1} \exp (-\delta / 2)\right)$. In this case the diquark condensation, or in other words, superconducting, phase is realized. The critical value $\mu_{c}$, at which the first order phase transition between these phases takes pace, can be easily calculated,

$$
\mu_{c}=M_{1} \sqrt{\frac{1-e^{-\delta}}{2}}
$$

It follows from (29) that at $\delta \rightarrow \infty$ we have $\mu_{c} \rightarrow M_{1} / \sqrt{2}$, i.e. even at arbitrary small interaction in the diquark channel there is a superconducting phase in the model at $\mu>\mu_{c}$. It is the so-called Cooper instability of the model.

Second, if $\delta<0$, then we have found only the diquark condensation phase for arbitrary values of $\mu$. It means that in the case, when an interaction in the diquark channel is stronger than in the quark-antiquark one the quark number $U_{V}(1)$ symmetry is spontaneously broken down and superconducting phase is formed in the model at $\mu>0$.

Finally, few words about the particle density $n=-\partial \Omega\left(M_{0}, \Delta_{0}\right) / \partial \mu$ of the system at zero temperature (where $\left(M_{0}, \Delta_{0}\right)$ is the GMP of the TDP (26) ). Suppose that $\delta>0$ and $\mu<\mu_{c}(29)$, i.e., that we are in the chiral symmetry breaking phase. Since in this case $M_{0}=M_{1}$ and $\Delta_{0}=0$, one can easily obtain from the expression (27) that $n \equiv 0$ in the chirally broken phase. However, at $\mu>\mu_{c}$ the system is in the superconducting phase, where $M_{0}=0$ and $\left.\Delta_{0}=M_{1} \exp (-\delta / 2)\right)$. As a result, it follows from the expression (28) that in this phase the particle density is nonzero, $n=\mu / \pi$. The last expression for particle density is also valid for superconducting phase at $\delta<0$.

The case $T \neq 0$. In order to include temperature into our consideration, let us start from the unrenormalized expression (14) for the TDP, where one should perform the following standard replacements:

$$
\int_{-\infty}^{\infty} \frac{d p_{0}}{2 \pi}(\cdots) \rightarrow i T \sum_{n=-\infty}^{\infty}(\cdots), \quad p_{0} \rightarrow p_{0 n} \equiv i \omega_{n} \equiv i \pi T(2 n+1), \quad n=0, \pm 1, \pm 2, \ldots
$$




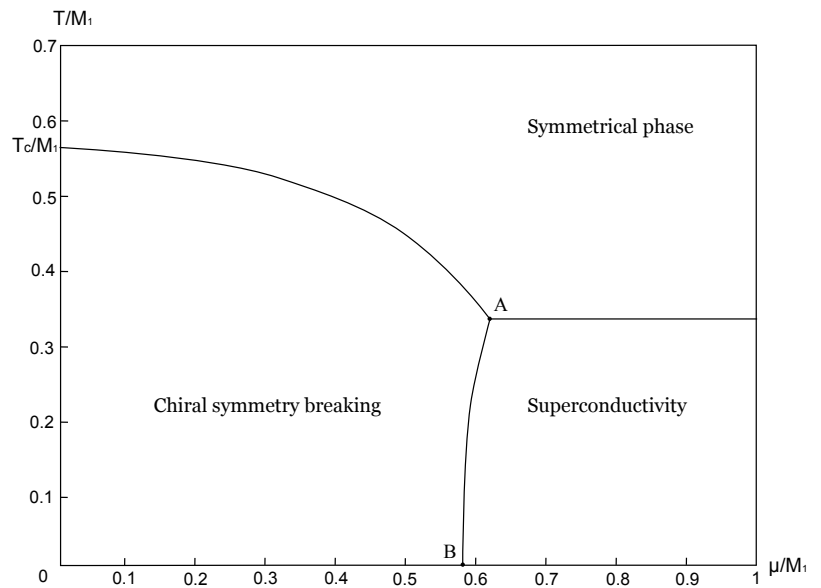

FIG. 1. The $(\mu, T)$-phase structure of the model at $\delta=1$ for the homogeneous case of the ansatz (8) for condensates $\left(b=b^{\prime}=0\right)$. Here $T_{c} \approx 0.57 M_{1}$. The $(\mu, T)$ coordinates of the points $\mathrm{A}$ and $\mathrm{B}$ are the following: $\mathrm{A} \equiv \mathrm{A}\left(0.62 M_{1}, 0.33 M_{1}\right), \mathrm{B} \equiv \mathrm{B}\left(0.59 M_{1}, 0\right)$. In the symmetrical phase $M=\Delta=0$.

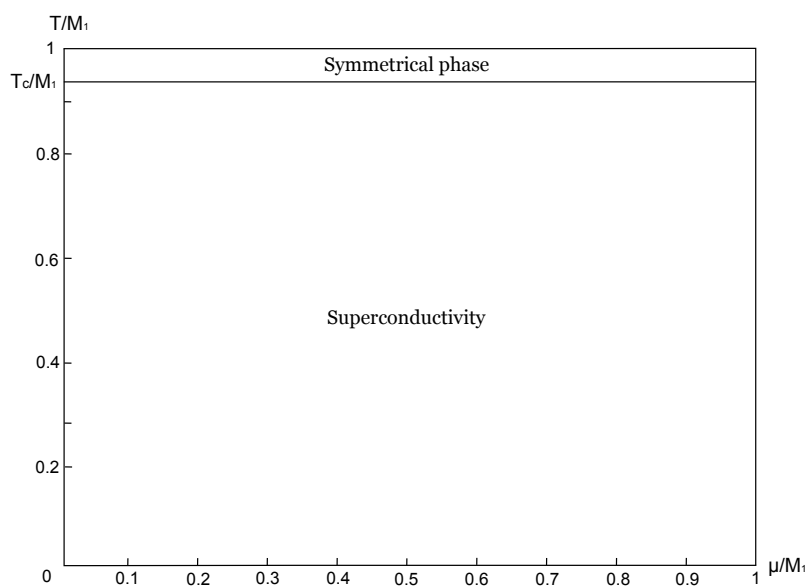

FIG. 2. The $(\mu, T)$-phase structure of the model at $\delta=-1$ both for homogeneous $\left(b=b^{\prime}=0\right)$ and inhomogeneous $\left(b \neq 0, b^{\prime} \neq 0\right)$ cases of the ansatz (8) for condensates. Here $T_{c} \approx 0.93 M_{1}$. In the symmetrical phase $M=\Delta=b=b^{\prime}=0$.

i.e. the $p_{0}$-integration should be replaced by the summation over an infinite set of Matsubara frequencies $\omega_{n}$. Summing over Matsubara frequencies in the obtained expression (the corresponding technique is presented, e.g., in [22]), one can find for the unrenormalized temperature dependent $\operatorname{TDP} \Omega_{T}^{u n}(M, \Delta)$ the following expression:

$$
\begin{aligned}
\Omega_{T}^{u n}(M, \Delta) & =\frac{M^{2}}{4 G_{1}}+\frac{\Delta^{2}}{4 G_{2}}-\int_{0}^{\infty} \frac{d p_{1}}{2 \pi}\left\{E_{+}+E_{-}\right\}-T \int_{0}^{\infty} \frac{d p_{1}}{\pi} \ln \left\{\left[1+e^{-\beta E_{+}}\right]\left[1+e^{-\beta E_{-}}\right]\right\} \\
& =\Omega^{u n}(M, \Delta)-T \int_{0}^{\infty} \frac{d p_{1}}{\pi} \ln \left\{\left[1+e^{-\beta E_{+}}\right]\left[1+e^{-\beta E_{-}}\right]\right\},
\end{aligned}
$$

where $\beta=1 / T, E_{ \pm}$are given in (15) and $\Omega^{u n}(M, \Delta)$ is just the unrenormalized TDP (16) at $T=0$. Since the last integral in (31) is convergent, in order to obtain a finite renormalized TDP at nonzero temperature, we should simply renormalize the TDP $\Omega^{u n}(M, \Delta)$, as it was done in the previous subsection III B. Thus, in the case of homogeneous condensates we obtain the following renormalized $T$ - and $\mu$-dependent TDP:

$$
\Omega_{T}(M, \Delta)=\Omega(M, \Delta)-T \int_{0}^{\infty} \frac{d p_{1}}{\pi} \ln \left\{\left[1+e^{-\beta E_{+}}\right]\left[1+e^{-\beta E_{-}}\right]\right\}
$$

where $\Omega(M, \Delta)$ is the renormalized TDP (26) at zero temperature and $\mu>0$. For particular values of $\delta=1$ and $\delta=-1$ the numerical investigations lead to the $(\mu, T)$-phase portraits shown in Fig. 1 and Fig. 2, respectively. Note that in Fig. 1, there is a phase transition of the first order on the boundary between the chiral symmetry breaking phase (in which $\Delta=0, M \neq 0$ ) and diquark, or superconducting, phase with $M=0, \Delta \neq 0$. However, on the boundary of the symmetrical phase there are second-order phase transitions in both figures. It is interesting also to look at the behavior of the particle density

$$
n=-\partial \Omega_{T}\left(M_{0}, \Delta_{0}\right) / \partial \mu
$$

of the system at nonzero temperature. In this formula $M_{0}$ and $\Delta_{0}$ stand for coordinates of the GMP of the TDP (32). (It is evident that $M_{0}$ and $\Delta_{0}$ are temperature dependent quantities.) Hence, in the chiral symmetry breaking phase, where $M_{0} \neq 0$ but $\Delta_{0}=0$, the particle density (33), denoted in this phase as $n_{C S B}$, looks like

$$
n_{C S B}(\mu, T)=\frac{\theta\left(\mu-M_{0}\right)}{\pi} \sqrt{\mu^{2}-M_{0}^{2}}+\frac{1}{\pi} \int_{0}^{\infty} d p_{1}\left\{\frac{\theta(E-\mu)}{1+e^{\beta(E-\mu)}}-\frac{\theta(\mu-E)}{1+e^{\beta(\mu-E)}}-\frac{1}{1+e^{\beta(E+\mu)}}\right\},
$$

where $E=\sqrt{p_{1}^{2}+M_{0}^{2}}$. Assuming in (34) that $M_{0}=0$, one can obtain the particle density $\left(\equiv n_{S Y M}\right)$ in the symmetric phase of the model

$$
n_{S Y M}(\mu, T)=\frac{\mu}{\pi}+\frac{1}{\pi} \int_{0}^{\infty} d p_{1}\left\{\frac{\theta\left(p_{1}-\mu\right)}{1+e^{\beta\left(p_{1}-\mu\right)}}-\frac{\theta\left(\mu-p_{1}\right)}{1+e^{\beta\left(\mu-p_{1}\right)}}-\frac{1}{1+e^{\beta\left(p_{1}+\mu\right)}}\right\} \equiv \frac{\mu}{\pi},
$$

i.e. in the symmetrical phase of the model the particle density does not depend on temperature. Finally, since in the superconducting phase $M_{0}=0$ and $\Delta_{0} \neq 0$, it is possible to obtain, after some manipulations, the following 
expression for the particle density $\left(\equiv n_{S C}\right)$ in this phase:

$$
n_{S C}(\mu, T)=\frac{\mu}{\pi}-\frac{1}{\pi} \int_{0}^{\infty} d p_{1}\left\{\frac{\mu+p_{1}}{\epsilon_{+}\left[1+e^{\beta \epsilon_{+}}\right]}+\frac{\mu-p_{1}}{\epsilon_{-}\left[1+e^{\beta \epsilon_{-}}\right]}\right\} \equiv \frac{\mu}{\pi} .
$$

In (36) we use the notations $\epsilon_{ \pm}=\sqrt{\Delta_{0}^{2}+\left(\mu \pm p_{1}\right)^{2}}$. It is clear that in the superconducting phase the particle density is also a temperature independent quantity.

It is evident that the plots of the functions $n_{C S B}(\mu, T), n_{S Y M}(\mu, T)$ and $n_{S C}(\mu, T)$ are some surfaces in three dimensional parametric space $(\mu, T, n)$. As a whole, the combination of these surfaces are no more than the plot of the particle density (33) $n(\mu, T)$ vs $\mu, T$. At $\delta=1$ it is depicted in Fig. 3. Recall, the $(\mu, T)$-phase portrait of the model was already presented in Fig. 1 at $\delta=1$. However, sometimes the phase diagram in terms of the fermion number density $n$ and temperature $T$ is more informative. To obtain the $(n, T)$-phase portrait of the model one should simply construct projections of the boundaries of the surfaces $n_{C S B}(\mu, T), n_{S Y M}(\mu, T)$ and $n_{S C}(\mu, T)$ (in Fig. 3 these boundaries are represented as thick solid lines) onto the $(n, T)$-coordinate plane. As a result, we will divide the $(n, T)$-plane into several different phases. 5 Performing this procedure in the case $\delta=1$ (see Fig. 3), it is possible to find the $(n, T)$-phase diagram at $\delta=1$. It is presented in Fig. 4, where you can see the usual (pure) chiral symmetry breaking, superconducting and symmetrical phases of the model. In addition, there is a region of the figure corresponding to a co-existence (or mixture) of the chiral symmetry breaking and superconducting phases. (The co-existence of two arbitrary phases means that in the space filled by one of them there are bubbles of another phase and vice versa.) In this case in the chiral symmetry breaking phase there might appear at some temperature a bubble of a more dense superconducting phase. If energy is provided to the system, then (at fixed value of a chemical potential) the size of the bubble is increased, i.e. the average particle density of the system is also increased. The process can be presented in Fig. 4 as a movement along the straight line parallel to the density axis, which crosses the region of the phase co-existence. Note also that the region of phase mixture in Fig. 4 is represented in Fig. 1 as a first-order phase transition curve AB (in Fig. 3 it corresponds to a vertical cylindrical surface, connecting the $n_{C S B}$ and $n_{S C}$ surfaces).

The above mentioned way for constructing $(n, T)$-phase diagram of the model can be used in the case $\delta<0$ as well. Qualitatively, it is the same as the phase portrait of Fig. 2, in which one should simply rename the horizontal $\mu$-axis in favor of $n$-axis.

It is clear from Figs 1,2 and 4 that in the case of homogeneous condensates the temperature of a chiral symmetry restoration phase transition depends strongly on values of chemical potential $\mu$ and/or particle density $n$. In contrast, the critical temperature $T_{\text {crit }}$ of a transition between symmetrical and superconducting phases does not depend on $\mu$ and/or $n$. However, in the framework of the model under consideration this property is valid only in the $(1+1)$ dimensional spacetime. In higher dimensions (see, e.g., the paper [20], where just the same model (1), but in the $(2+1)$-dimensional spacetime, was investigated) the critical temperature of a transition between symmetrical and superconducting phases is already a $\mu$-dependent quantity. The conclusion is also supported by results of the paper [24], where the phase structure of a (3+1)-dimensional version of the model (1) was considered. In [24], one coordinate was compactified ( $L$ is the radius of a compactification). It was shown there that in the case of antiperiodic boundary conditions for fermion fields the critical value of $1 / L$, at which superconductivity is transformed into symmetric phase, depends on $\mu$ (see Fig. 9 of [24]). Since at antiperiodic boundary conditions the quantity $1 / L$ behaves in many respects like temperature, one can believe that in the framework of the (3+1)-dimensional model (1) $T_{\text {crit }}$ also depends on $\mu$.

\section{INHOMOGENEOUS CASE OF THE ANSATZ (8) FOR CONDENSATES: $b \neq 0, b^{\prime} \neq 0$}

In this section we determine all quantities entering the ansatz (8) for condensates dynamically, i.e. we search for $\Delta, M, b$, and $b^{\prime}$ taking into account the stationarity equations for the TDP (12). In particular, it means that from the very beginning the quantities $b$ and $b^{\prime}$ are not supposed to be zero a priory (as in the previous section). In this case the TDP (12) of the system at $T=0$ can be transformed to the following expression:

$$
\begin{aligned}
\Omega\left(M, b, b^{\prime}, \Delta\right) & =\frac{M^{2}}{4 G_{1}}+\frac{\Delta^{2}}{4 G_{2}}+\frac{i}{2} \int \frac{d^{2} p}{(2 \pi)^{2}} \ln \left[\Delta^{4}-2 \Delta^{2}\left(p_{0}^{2}-p_{1}^{2}+M^{2}+b^{\prime 2}-\tilde{\mu}^{2}\right)\right. \\
& \left.+\left(M^{2}+\left(p_{1}-b^{\prime}\right)^{2}-\left(p_{0}-\tilde{\mu}\right)^{2}\right)\left(M^{2}+\left(p_{1}+b^{\prime}\right)^{2}-\left(p_{0}+\tilde{\mu}\right)^{2}\right)\right],
\end{aligned}
$$

where $\tilde{\mu}=\mu-b$. The expression (37) of the TDP resembles the thermodynamic potential of the (1+1)-dimensional model with inhomogeneous charged pion condensation phenomenon (see, e.g., the expression (17) in [14], where the corresponding TDP is presented). Thus, using the same rather tedious technique as in [14], it is possible to show that at arbitrary fixed $M, b, \Delta$ the absolute minimum of the function (37) vs $b^{\prime}$ always occurs at $b^{\prime}=0$. This means

\footnotetext{
${ }^{5}$ It is evident that one reproduces the $(\mu, T)$-phase portrait of Fig. 1, when finds the projections of all boundaries of the above mentioned surfaces onto the $(\mu, T)$-coordinate plane of Fig. 3.
} 


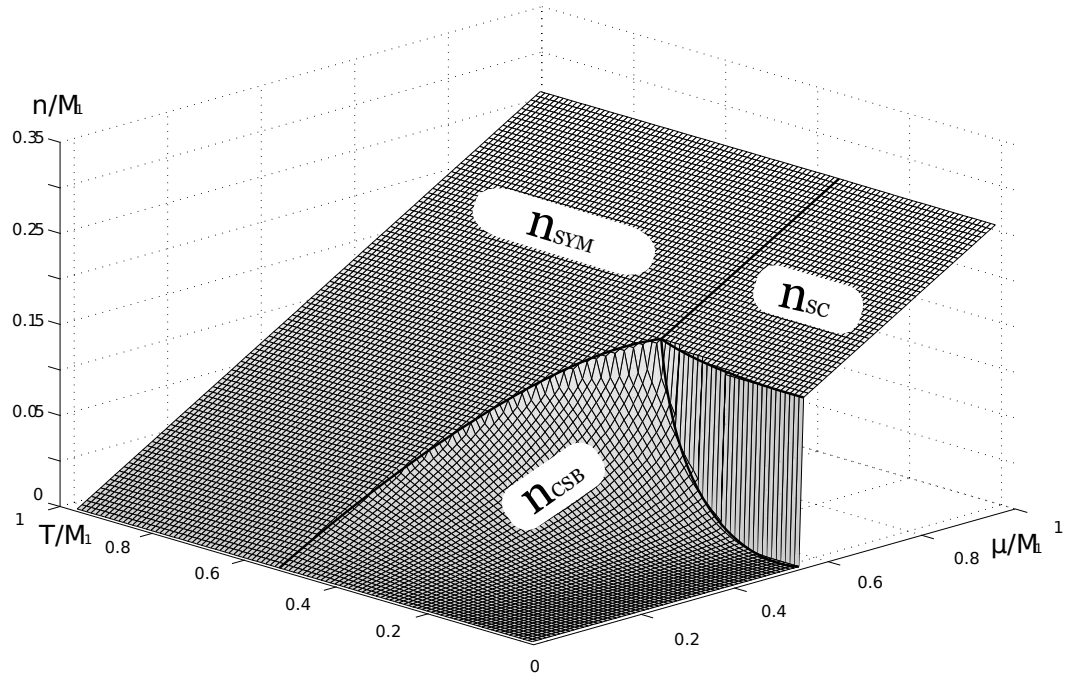

FIG. 3. The particle density $n$ vs $(\mu, T)$ at $\delta=1$ in the homogeneous case of the ansatz (8) for condensates $\left(b=b^{\prime}=0\right)$. The surfaces $n_{C S B}, n_{S Y M}$ and $n_{S C}$ represent the behavior of the particle density $n(\mu, T)$ (33) in the chiral symmetry breaking-, symmetrical- and superconducting phases of the model, respectively.



FIG. 4. Phase structure of the model in terms of particle density $n$ and temperature $T$ at $\delta=1$ in the homogeneous case of the ansatz (8) for condensates $\left(b=b^{\prime}=0\right)$.

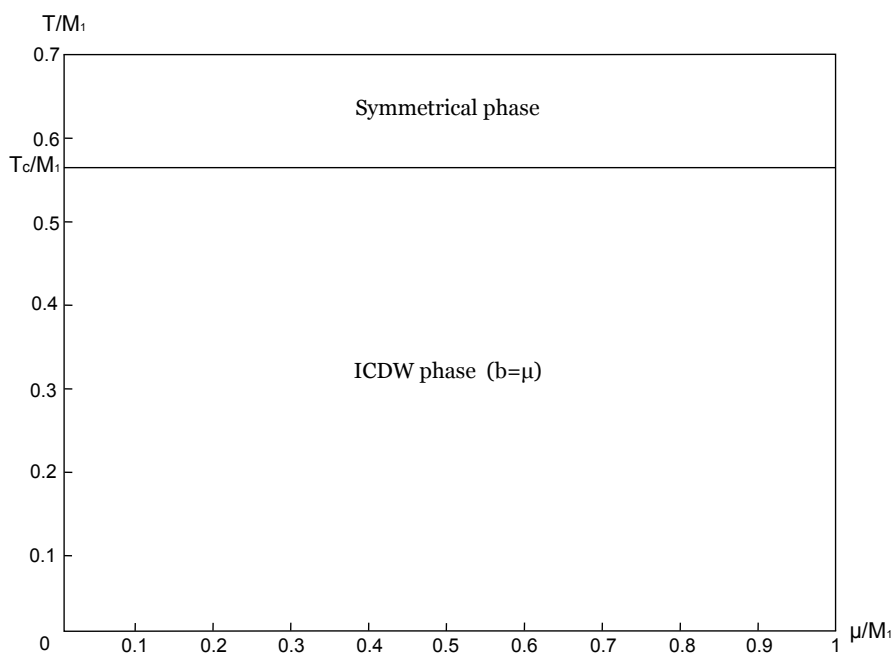

FIG. 5. $(\mu, T)$-phase structure of the model at $\delta=1$, when the possibility for condensate inhomogeneity in the framework of the ansatz (8) is taken into account. At $T<T_{c} \approx 0.57 M_{1}$ the inhomogeneous chiral density wave (ICDW) phase is arranged, where we have for condensates: $\Delta=0, b^{\prime}=0, M \geq 0$, and $b=\mu$. In the symmetrical phase $\Delta=b^{\prime}=M=b=0$.

that in the framework of the ansatz (8), the diquark condensate is always spatially homogeneous within the model (1). Now, taking into account this result, we suppose that $b^{\prime}=0$ in what follows. 6 As a consequence, in this case the unrenormalized TDP (12) looks like the expressions (14)-(16) in which $\mu$ should be replaced by $\tilde{\mu} \equiv \mu-b$, i.e.

$$
\left.\Omega^{u n}(M, b, \Delta) \equiv \Omega^{u n}\left(M, b, b^{\prime}, \Delta\right)\right|_{b^{\prime}=0}=\frac{M^{2}}{4 G_{1}}+\frac{\Delta^{2}}{4 G_{2}}-\frac{1}{2 \pi} \int_{0}^{\infty} d p_{1} \tilde{E}_{+}-\frac{1}{2 \pi} \int_{0}^{\infty} d p_{1} \tilde{E}_{-},
$$

where

$$
\tilde{E}_{ \pm}^{2}=\tilde{\mu}^{2}+p_{1}^{2}+M^{2}+\Delta^{2} \pm 2 \sqrt{M^{2} \Delta^{2}+\tilde{\mu}^{2}\left(p_{1}^{2}+M^{2}\right)}
$$

In contrast to the TDP (16) of the previous section, the TDP (38) is a function of three variables, $M, \Delta$ and $b$. Since, in this case $b$ simply shifts effectively the chemical potential $\mu$, one can consider the TDP (38) as a function of $M, \Delta$

\footnotetext{
${ }^{6}$ To avoid overloading of our paper by extra formulas, we omit a detailed proof of this fact. Moreover, earlier in the paper [23] the same result was obtained in the particular case of the model (1) with $G_{1}=0$.
} 
and $\tilde{\mu}=\mu-b$. Clearly, without loss of generality one can study the expression (38) in the region $M \geq 0, \Delta \geq 0$ and $\tilde{\mu} \geq 0$.

To find a finite renormalized expression for the TDP (38), we should first regularize it and then perform a renormalization procedure in order to remove at $\Lambda \rightarrow \infty$ the UV divergences by demanding an appropriate behavior of the bare coupling constants $G_{1,2}$ vs the cutoff parameter $\Lambda$. In the case of spatially homogeneous condensates all regularization schemes are usually equivalent. However, in the case of spatially inhomogeneous condensates the translational invariance over one or several spatial coordinates is lost. So, the corresponding (spatial) momenta are not conserved. Then, if one uses the momentum-cutoff regularization technique, as in the previous section, nonphysical (spurious) $b$-dependent terms appear, and the TDP acquires some non-physical properties such as unboundedness from below with respect to $b$, etc. In order to obtain a physically reliable TDP (or effective potential), in this case an additional substraction procedure is usually applied (for details see in [7, 13]). On the other hand, if one uses more adequate regularization schemes such as Schwinger proper-time [2, 3] or energy-cutoff regularizations [8, 14], etc., such spurious terms do not appear. 7

In the present paper the slightly modified energy cutoff regularization scheme of [8] is adopted. (See also [13, 23], where a similar regularization was used in searching for both chiral density waves and inhomogeneous diquark condensate in some $\mathrm{NJL}_{2}$ models.) Namely, we require that only energies with momenta $p_{1}$, constrained by the relations $\tilde{E}_{ \pm}(M=0, \Delta=0)=p_{1} \pm \tilde{\mu}<\Lambda$, contribute to the regularized thermodynamic potential. This means that the term with energy $\tilde{E}_{+}\left(\tilde{E}_{-}\right)$in (38) should be integrated in the regularized expression for TDP over the interval $0<p_{1}<\Lambda-\tilde{\mu}\left(0<p_{1}<\Lambda+\tilde{\mu}\right)$. Consequently, instead of (38) we have the following regularized TDP:

$$
\Omega^{r e g}(M, b, \Delta)=\frac{M^{2}}{4 G_{1}}+\frac{\Delta^{2}}{4 G_{2}}-\frac{1}{2 \pi} \int_{0}^{\Lambda-\tilde{\mu}} d p_{1} \tilde{E}_{+}-\frac{1}{2 \pi} \int_{0}^{\Lambda+\tilde{\mu}} d p_{1} \tilde{E}_{-},
$$

The expression (40) can be presented in the following form:

$$
\Omega^{r e g}(M, b, \Delta)=\widetilde{\Omega}^{r e g}(M, b, \Delta)+\frac{1}{2 \pi} \int_{\Lambda-\tilde{\mu}}^{\Lambda} d p_{1} \tilde{E}_{+}-\frac{1}{2 \pi} \int_{\Lambda}^{\Lambda+\tilde{\mu}} d p_{1} \tilde{E}_{-},
$$

where $\widetilde{\Omega}^{r e g}(M, b, \Delta)$ is the TDP (17) with simple replacement $\mu \rightarrow \tilde{\mu}$. Replacing in this formula $G_{1,2}$ by $G_{1,2}(\Lambda)$ from (201) and adding an unessential constant $\Lambda^{2} / 2 \pi$, we obtain in the limit $\Lambda \rightarrow \infty$ the renormalized expression

$$
\Omega(M, b, \Delta)=\widetilde{\Omega}(M, b, \Delta)+\frac{\tilde{\mu}^{2}}{2 \pi}-\frac{\mu^{2}}{2 \pi},
$$

where

$$
\widetilde{\Omega}(M, b, \Delta)=V_{0}(M, \Delta)-\int_{0}^{\infty} \frac{d p_{1}}{2 \pi}\left(\tilde{E}_{+}+\tilde{E}_{-}-\sqrt{p_{1}^{2}+(M+\Delta)^{2}}-\sqrt{p_{1}^{2}+(M-\Delta)^{2}}\right)
$$

and $V_{0}(M, \Delta)$ is given in (24). Note that the TDP (43) is formally equal to the renormalized TDP (26) of the homogeneous condensates case, in which one should simply perform the replacement $\mu \rightarrow \tilde{\mu}$. To obtain the last terms in (42), one should take into account that at $\Lambda \rightarrow \infty$ the $p_{1}$-values in both integrals in (41) are much greater than $M, \Delta, b, \mu$. In this case, it is possible to expand the quantities $\tilde{E}_{ \pm}$into power series of $p_{1}$ and then to integrate each term. Moreover, we add in the expression (42) an unessential $b$-independent term, $-\mu^{2} / 2 \pi$, in order to reproduce at $b=0$ the TDP (26), corresponding to a spatially homogeneous chiral condensate.

Phase structure at $T=0$. It is clear that to find the phase portrait of the model at $T=0$, one should investigate the global minimum point (GMP) of the TDP $\Omega(M, b, \Delta)$ (42) vs the dynamical variables $M, b, \Delta$. Since in our case the variable $b$ is absorbed by the chemical potential, the TDP (42) is indeed a function of three variables $M, \Delta, \tilde{\mu} \equiv \mu-b$. Thus, searching for the GMP of this function consists effectively of two stages. First, one can find the extremum of this function over $M$ and $\Delta$ (taking into account the results of section [IIB) 8 and then one minimizes the obtained expression over the variable $\tilde{\mu}$. Following this strategy, let us introduce the quantity

$$
\Omega(\tilde{\mu})=\min _{M \geq 0, \Delta \geq 0}\{\Omega(M, b, \Delta)\} .
$$

Now suppose that $\delta>0$. Taking into account the results of the investigation of the GMP of the TDP (26) (see subsection (IIB), it is easy to see that at $\tilde{\mu}<\mu_{c}$ with $\mu_{c}$ given in (29), the function $\Omega(M, b, \Delta)$ is minimized at $M=M_{1}, \Delta=0$ (recall, $\tilde{\mu}$ is fixed). In this case $4 \pi \Omega(\tilde{\mu})=-M_{1}^{2}-2 \mu^{2}+2 \tilde{\mu}^{2}$. However, at $\tilde{\mu}>\mu_{c}$ the minimum of

\footnotetext{
${ }^{7}$ As discussed in the recent papers [2, 3, 8, 13, 14], an adequate regularization scheme in the case of spatially inhomogeneous phases consists in the following: for different quasiparticles the same restriction on their region of energy values $\tilde{E}_{ \pm}$should be used in a regularized thermodynamic potential.

8 As in the case with $b=0$, in the inhomogeneous case we did not find local minima of the TDP (42) in which both $M \neq 0$ and $\Delta \neq 0$.
} 
$\Omega(M, b, \Delta)$ is reached at $M=0, \Delta=M_{1} \exp (-\delta / 2)$. Hence, in this case $4 \pi \Omega(\tilde{\mu})=-M_{1}^{2} \exp (-\delta)-2 \mu^{2}$. Therefore, the function (44) takes the form

$$
4 \pi \Omega(\tilde{\mu})=\left\{\begin{array}{lll}
-M_{1}^{2}-2 \mu^{2}+2 \tilde{\mu}^{2}, & \text { if } \quad \tilde{\mu}<\mu_{c} \\
-M_{1}^{2} \exp (-\delta)-2 \mu^{2}, & \text { if } \quad \tilde{\mu}>\mu_{c} .
\end{array}\right.
$$

Since for arbitrary fixed $\mu>0$ the minimum of the function (45) over the variable $\tilde{\mu} \geq 0$ occurs at $\tilde{\mu}=0$, i.e. at $b=\mu$, we conclude that for arbitrary values of $\delta>0$ and $\mu>0$ the spatially inhomogeneous phase in the form of chiral spirals (chiral density waves) is more preferable than either of the two homogeneous phases, homogeneous chiral symmetry breaking phase or homogeneous phase with nonzero diquark condensate.

In the case of $\delta<0$ the GMP of the TDP $\Omega(M, b, \Delta)$ over two variables, $M$ and $\Delta$, lies at the point $(M=0, \Delta=$ $M_{1} \exp (-\delta / 2)$ ) (see subsection $\amalg$ IIB). Hence, in this case $4 \pi \Omega(\tilde{\mu})=-M_{1}^{2} \exp (-\delta)-2 \mu^{2}$, i.e. it does not depend on $\tilde{\mu}$. As a result, we could take $b=0$ which corresponds to the homogeneous diquark condensation phase at arbitrary $\delta<0$ and $\mu>0$ values, i.e. the (homogeneous) superconductivity phenomenon can appear in the model only at $G_{2}>G_{1}$. In the case $G_{2}<G_{1}$, it is suppressed by inhomogeneous chiral density wave phase. In contrast, if the homogeneous ansatz for condensates is used (see [17], as well as the previous section), then Cooper pairing appears in the model at arbitrary $G_{2}<G_{1}$ starting from $\mu>\mu_{c}$ (29).

Phase structure at $T \neq 0$. In order to include the temperature into consideration, let us start from the unrenormalized expression (12) for the TDP. Then at $b^{\prime}=0$ we have

$$
\Omega^{u n}(M, b, \Delta)=\frac{M^{2}}{4 G_{1}}+\frac{\Delta^{2}}{4 G_{2}}+\frac{i}{2} \int \frac{d^{2} p}{(2 \pi)^{2}} \ln \left[\left(p_{0}^{2}-\tilde{E}_{+}^{2}\right)\left(p_{0}^{2}-\tilde{E}_{-}^{2}\right)\right] .
$$

(Obviously, the TDP (46) is equal to the expression (38).) To get the corresponding unrenormalized thermodynamic potential $\Omega_{T}^{u n}(M, b, \Delta)$ in the case of nonzero temperature, one again performs the standard replacements (30) in (46). Summing over Matsubara frequencies in the obtained expression, one finds

$$
\begin{aligned}
\Omega_{T}^{u n}(M, b, \Delta) & =\frac{M^{2}}{4 G_{1}}+\frac{\Delta^{2}}{4 G_{2}}-\int_{0}^{\infty} \frac{d p_{1}}{2 \pi}\left\{\tilde{E}_{+}+\tilde{E}_{-}\right\}-T \int_{0}^{\infty} \frac{d p_{1}}{\pi} \ln \left\{\left[1+e^{-\beta \tilde{E}_{+}}\right]\left[1+e^{-\beta \tilde{E}_{-}}\right]\right\} \\
& =\Omega^{u n}(M, b, \Delta)-T \int_{0}^{\infty} \frac{d p_{1}}{\pi} \ln \left\{\left[1+e^{-\beta \tilde{E}_{+}}\right]\left[1+e^{-\beta \tilde{E}_{-}}\right]\right\}
\end{aligned}
$$

where $\beta=1 / T, \tilde{E}_{ \pm}$are given in (39), and $\Omega^{u n}(M, b, \Delta)$ is the unrenormalized TDP (38) at $T=0$. Since the last integral in (47) is convergent, we should again renormalize the term $\Omega^{u n}(M, b, \Delta)$ using the same regularization scheme as above in this section (see expression (40) for the corresponding regularized TDP), in order to obtain a finite renormalized TDP $\Omega_{T}(M, b, \Delta)$ at nonzero temperature. As a result, we have

$$
\Omega_{T}(M, b, \Delta)=\Omega(M, b, \Delta)-T \int_{0}^{\infty} \frac{d p_{1}}{\pi} \ln \left\{\left[1+e^{-\beta \tilde{E}_{+}}\right]\left[1+e^{-\beta \tilde{E}_{-}}\right]\right\},
$$

where $\Omega(M, b, \Delta)$ is given in (42). After numerical investigations of this TDP we obtain, e.g., at $\delta=1$ the $(\mu, T)$-phase portrait in Fig. 5. It is clear from this figure that at rather low temperatures $T<T_{c}$ and arbitrary values of chemical potential, the chiral symmetry breaking phase with inhomogeneous condensate in the form of chiral spiral is arranged (in Fig. 5 it is denoted as inhomogeneous chiral density wave (ICDW) phase). In this phase $\Delta=0, M \neq 0$, and wave vector $b$ does not depend on $T$ and equals $\mu$ (at each fixed value of $\mu$ the gap $M$ decreases in this phase continuously from $M_{1}$ to zero, when temperature varies from zero to $T_{c}$ ).

If $\delta<0$, then in the framework of the ansatz (8) we have not found any phase with nonzero values of $b$ and/or $b^{\prime}$, so in this case the $(\mu, T)$-phase portrait of the model is the same as in Fig. 2.

Finally, a few words about the particle density behavior as well as the density-temperature phase portrait of the model, when spatial inhomogeneity of condensates in the form of the ansatz (8) is allowed. In this case we denote the particle density of the model by $\tilde{n}$,

$$
\tilde{n}=-\partial \Omega_{T}\left(M_{0}, b_{0}, \Delta_{0}\right) / \partial \mu,
$$

where the TDP $\Omega_{T}(M, b, \Delta)$ is given in (48) and $M_{0}, b_{0}, \Delta_{0}$ are the coordinates of its global minimum point. It is evident that in the symmetrical phase of the model, where $\Delta_{0}=M_{0}=b_{0}=0$, the particle density (49) is equal to expression (35), i.e. $\tilde{n}_{S Y M}=\mu / \pi$.

In contrast, in the ICDW phase (see Fig. 5) we have $b_{0}=\mu, \Delta_{0}=0$, and $M_{0} \neq 0$ (the last quantity is a $T$-dependent one). So at $b=\mu$ the integral term of the expression (48) does not depend on $\mu$ and, hence, does not contribute to the particle density (49) in the ICDW phase. Moreover, as it follows from the expressions (42) and (43), in this case, i.e. at $b=\mu$, the TDP $\Omega(M, b, \Delta)$ in (48) can be presented in the form: $\Omega(M, b=\mu, \Delta)=-\mu^{2} /(2 \pi)+\cdots$, where we have omitted all the $\mu$-independent terms. As a result, it is easy to see from (49) that inside of the ICDW phase the particle density $\left(\equiv \tilde{n}_{I C D W}\right.$ ) does not depend on $T$ and, furthermore, $\tilde{n}_{I C D W}=\mu / \pi$. It clear from this discussion that the particle density-temperature phase diagram of the model has a rather trivial form, when the inhomogeneity 
ansatz (8) for condensates is applied. Indeed, one should take the $(\mu, T)$-phase portrait (it is Fig. 5 at $\delta>0$ or Fig. 2 at $\delta<0)$ and then simply rename the horizontal $\mu$-axis in favor of $\tilde{n}$-axis.

It is clear from the above consideration that in the case of inhomogeneous condensates the critical temperature, at which there occurs a symmetry restoring phase transition, does not depend on $\mu$ and/or $\tilde{n}$. However, it is a model dependent effect. Indeed, in this paper we deal with the model (1) which is invariant with respect to $U_{A}(1)$-chiral symmetry. In contrast, in (1+1)-dimensional model with $S U_{A}(2)$-chiral symmetry [13] the temperature of a transition between chiral density wave- and symmetrical phases is a $\mu$-dependent quantity. Moreover, in the last model the phase diagram has a more complicated form (see [13] for details).

\section{CONCLUSIONS}

We have investigated the phase structure of the $\mathrm{NJL}_{2}$-type model (1) in the framework of the Fulde-Ferrel single plane wave spatially non-uniform ansatz (8) both for chiral and superconducting condensates. The following results are obtained:

1) It is shown that spatially inhomogeneous superconducting condensation is forbidden in the model (1) (of course, in the framework of the ansatz (8) ).

2) It is clear from Fig. 5 that in the case $G_{1}>G_{2}$ the inhomogeneous chiral condensate in the form of the so-called chiral density wave suppresses the appearance of the superconductivity at arbitrary values of $\mu>0$. In contrast, if chiral- and Cooper condensates are assumed to be spatially uniform, then at sufficiently high $\mu$ the superconducting phase is allowed to exist in this case (see Fig. 1 and [17]).

3) If spatially inhomogeneous ansatz (8) for condensates is taken into account in the model (1), then Cooper pairing (which is spatially homogeneous) is possible only at sufficiently strong interaction in the quark-quark channel, i.e. at $G_{2}>G_{1}$ (see Fig. 2).

[1] D.V. Deryagin, D.Y. Grigoriev and V.A. Rubakov, Int. J. Mod. Phys. A 7, 659 (1992); M. Sadzikowski and W. Broniowski, Phys. Lett. B 488, 63 (2000); W. Broniowski, Acta Phys. Polon. Supp. 5, 631 (2012).

[2] E. Nakano and T. Tatsumi, Phys. Rev. D 71, 114006 (2005).

[3] D. Nickel, Phys. Rev. D 80, 074025 (2009); S. Carignano, D. Nickel and M. Buballa, Phys. Rev. D 82, 054009 (2010); H. Abuki, D. Ishibashi and K. Suzuki, arXiv:1109.1615.

[4] S. Maedan, Prog. Theor. Phys. 123, 285 (2010); A. Flachi, JHEP 1201, 023 (2012); arXiv:1304.6880 [hep-th].

[5] A. Heinz, arXiv:1301.3430 [hep-ph].

[6] T. Kojo, Y. Hidaka, L. McLerran and R.D. Pisarski, Nucl. Phys. A 843, 37 (2010).

[7] E.V. Gorbar, M. Hashimoto and V.A. Miransky, Phys. Rev. Lett. 96, 022005 (2006); J.O. Andersen and T. Brauner, Phys. Rev. D 81, 096004 (2010); C.f. Mu, L.y. He and Y.x. Liu, Phys. Rev. D 82, 056006 (2010).

[8] I.E. Frolov, K.G. Klimenko and V.Ch. Zhukovsky, Phys. Rev. D 82, 076002 (2010); Moscow Univ. Phys. Bull. 65, 539 (2010).

[9] E.J. Ferrer, V. de la Incera and A. Sanchez, arXiv:1205.4492.

[10] V. Schon and M. Thies, Phys. Rev. D 62, 096002 (2000); A. Brzoska and M. Thies, Phys. Rev. D 65, 125001 (2002).

[11] O. Schnetz, M. Thies and K. Urlichs, Annals Phys. 314, 425 (2004); G. Basar, G.V. Dunne and M. Thies, Phys. Rev. D 79, 105012 (2009); C. Boehmer and M. Thies, Phys. Rev. D 80, 125038 (2009); J. Hofmann, Phys. Rev. D 82, 125027 (2010).

[12] F. Correa, G.V. Dunne and M.S. Plyushchay, Annals Phys. 324, 2522 (2009).

[13] D. Ebert, N.V. Gubina, K.G. Klimenko, S.G. Kurbanov, V.C. Zhukovsky, Phys. Rev. D84, 025004 (2011).

[14] N.V. Gubina, K.G. Klimenko, S.G. Kurbanov and V.C. Zhukovsky, Phys. Rev. D 86, 085011 (2012).

[15] N.V. Gubina, K.G. Klimenko, S.G. Kurbanov and V.C. Zhukovsky, Moscow Univ. Phys. Bull. 67, 131 (2012); Yad. Fiz. 76, 1443 (2013).

[16] H. Caldas and M. A. Continentino, J. Phys. B: At. Mol. Opt. Phys. 46, 155301 (2013); D. Roscher, J. Braun and J.E. Drut, arXiv:1311.0179 [cond-mat.quant-gas].

[17] A. Chodos, H. Minakata, F. Cooper, A. Singh, and W. Mao, Phys. Rev. D 61, 045011 (2000).

[18] P. Fulde and R.A. Ferrel, Phys. Rev. 135, A550 (1964).

[19] K. Fujikawa, Phys. Rev. D 21, 2848 (1980).

[20] K.G. Klimenko, R.N. Zhokhov and V.C. Zhukovsky, Phys. Rev. D 86, 105010 (2012).

[21] D. Ebert, K.G. Klimenko, Phys. Rev. D80, 125013 (2009).

[22] L. Jacobs, Phys. Rev. D 10, 3956 (1974); K.G. Klimenko, Theor. Math. Phys. 70,87 (1987).

[23] K. Ohwa, Phys. Rev. D 65, 085040 (2002).

[24] D. Ebert and K.G. Klimenko, Phys. Rev. D 82, 025018 (2010). 